\title{
JUSTIFICATION OF PARAMETERS OF VACUUM PUMPING MEANS WITH PULSATING ACTIVE FLOW FOR CATTLE FARMS
}

\author{
Insaf Nafikov, Rail Khusainov, Ruslan Lukmanov, Ilgiz Galiyev \\ Kazan State Agrarian University, Russia \\ insaf-82@mail.ru,rail-1312@mail.ru, look-rus@mail.ru,drgali@mail.ru
}

\begin{abstract}
Annotation. One of the main components of the technological processes of animal husbandry are vacuum pumping means designed for dosing, mixing, transportation, feed preparation and feed distribution lines, milking cows, milk processing, as well as a large number of technological processes in agriculture. The article deals with a pulsating jet vacuum pumping device, widely used in all industries and agriculture, the feature of which is to improve the parameters of the technical characteristics of the pump without direct expenditure of mechanical energy with a simple design. In pulsating jet vacuum pumping devices, non-stationary modes are created intentionally to increase the ejection coefficient, productivity, etc. The pulsating flows of the transported materials are very diverse, this is due to the increased number of similarity numbers that determine their mode. If the reduced velocity and the Reynolds number are usually used for a stationary flow, for a pulsating flow, the relative frequency and the relative amplitude of the pulsations are added to them. The objects of experimental research were ejectors with pulsating motion of the active flow. The main task of the experiments was to determine the most effective indicators of the ejectors, such as the degree of pressure increase, the ejection coefficient and the geometric parameter. As a result of the experiment, the regularities of changes in the performance of a pulsating jet vacuum pumping device were obtained depending on the valve material and the magnitude of its pulsation. It is revealed that for an ejector with a pulsating movement of the active flow, the recommended valve material is a metal ball, with a pulsation frequency of $90 \ldots 100 \mathrm{~min}^{-1}$. As a result of the application of the proposed ejector with pulsating movement of the active flow, the transportation capacity will increase by $14.5 \%$.
\end{abstract}

Keywords: vacuum, ejector, pulsating flow, pulsator, nozzle, mixing chamber.

\section{Introduction}

An integral part of the agro-industrial complex is the production of high-quality agricultural products, which is associated with the mechanization of technological processes [1-4]. Currently, work is underway to find effective technical solutions to improve these processes [5-8].

One of the main components of the technological processes of animal husbandry is vacuum pumping means designed for dosing, mixing, transportation, drying, feed preparation and feed distribution lines, milking cows, milk processing, as well as a large number of technological processes in agriculture $[9 ; 10]$. Among the disadvantages of the existing vacuum pumping facilities, it can be noted that the output parameters are limited by the technical characteristics of the pump [11-13].

The study found that one of the ways to increase the efficiency of vacuum pumping means for mixing, dosing of gaseous media and bulk materials, is intermittent (pulsating) the current of the active flow [14].

The use of pulsating jet vacuum pumping devices (ejectors) allows, along with reducing the residual pressure, to significantly reduce energy costs with the same dimensions. The use of non-stationary flows contributes to a more efficient energy exchange between the active and passive environment.

The effectiveness of the method of influencing the medium, the parameters of which depend on time, can be explain by the possibility of transferring motion from the active medium to the passive one using pressure waves, as well as by organizing the process with sequential and parallel addition of additional mass [14].

The analysis of the efficiency of ejectors with active non-stationary flows in comparison with the same ejectors with stationary flows showed an increase in the ejection coefficient by 1.5-2 times [14].

\section{Materials and methods}

Pulsation of the active medium supply to the mixing chamber can be achieved by periodically opening and closing the nozzle passage section, which is carried out by means of regulating elements that perform reciprocating motion (valve-type ejectors), as well as rotational motion (curtain-slot and drum-type devices). The most economically feasible in the conditions of agricultural production are valve-type ejectors (Figure 1) [15]. 


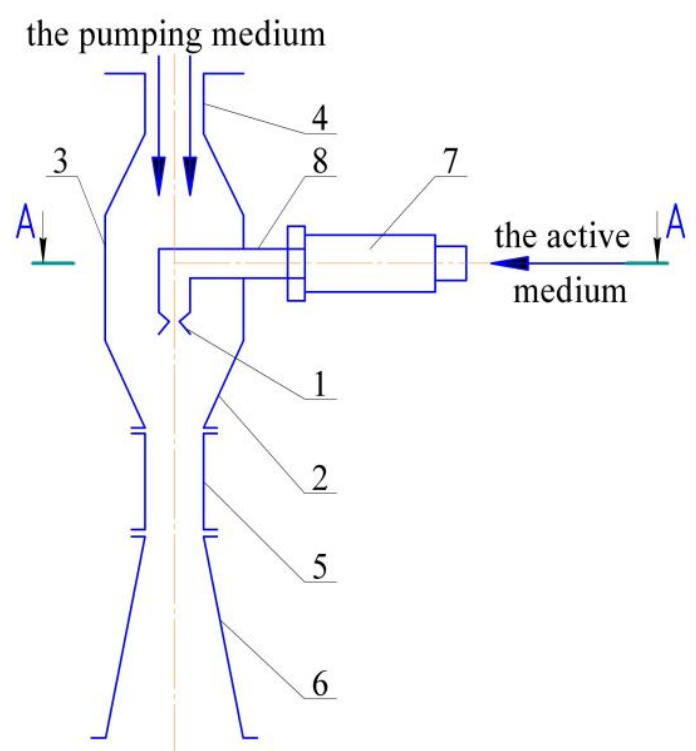

a)

A-A

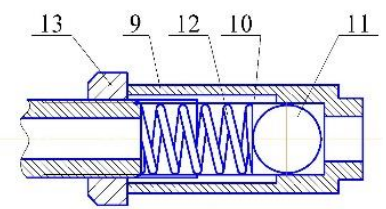

b)

A-A

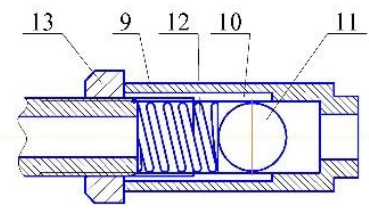

Fig. 1. Diagram of the pulsating ejector in composition of the water-ring vacuum pump (a - interrupting mechanism at the time of locking the supply of the active medium;

b - interrupting mechanism at the supply of the active medium): 1 - high-pressure nozzle;

2 - pumped medium; 3 - receiving chamber; 4 - supply pipe of the pumped medium; 5 - mixing chamber; 6 - diffuser; 7 - interrupting mechanism; 8 -inlet pipe;

9 - water-ring vacuum pump

The pulsating ejector includes a high-pressure nozzle 1 for supplying the active medium, made either of a tapering or tapering - expanding shape (depending on the aggregate state of the active medium). In the active medium supply line, an interrupting mechanism is installed in front of the central active nozzle, which consists of a cylindrical sleeve, where channels are provided for the supply of highpressure medium (Figure $1 \mathrm{a}, 1 \mathrm{~b}$ ). To ensure the pulsation of the high-pressure flow, a ball and a spring are provided inside the sleeve. The opening of the inlet port is carried out by the action of a high-pressure flow on the ball. The inlet opening is locked by the same ball due to the force action of a cylindrical spring, the elasticity of which is regulated by the installation of the sleeve by screwing it on the inlet pipe of the jet device.

To justify the design parameters of the pulsator, it is necessary to obtain the regularity of the influence of the pulsation frequency on the performance of the technological process. In the general case, for consideration of motion, the one-dimensional nonstationary equations of gas dynamics include the equations of flow-continuity (1), conservation of momentum (2), and energy (3) [16].

$$
\begin{gathered}
\frac{\partial}{\partial \tau}(\rho S)+\frac{\partial}{\partial x}(\rho V S)=0, \\
\frac{\partial}{\partial \tau}(\rho V S)+\frac{\partial}{\partial x}\left[S\left(\rho V^{2}+p\right)\right]=p \frac{\partial S}{\partial x}-\frac{\partial}{\partial x} \Delta I, \\
\frac{\partial}{\partial \tau}\left[\rho S\left(e+\frac{V^{2}}{2}\right)\right]+\frac{\partial}{\partial x}\left[\rho V S\left(e+\frac{p}{\rho}+\frac{V^{2}}{2}\right)\right]=0,
\end{gathered}
$$

where $\tau$-time, min;

$x$ - longitudinal coordinate, $\mathrm{m}$;

$\rho$ - gas density, $\mathrm{kg} \cdot \mathrm{m}^{-3}$;

$p$ - pressure, $\mathrm{kPa}$;

$V$ - flow rate, $\mathrm{m} \cdot \mathrm{s}^{-1}$;

$S$ - cross-sectional area of the channel, $\mathrm{m}^{2}$;

$e$ - base of the natural logarithm, $e=2.73$;

$\Delta I-$ friction energy losses of the gas and local losses, $\mathrm{kJ}$. 
The time variation of the static pressure $p(t)$, the flow rate or pressure regulation is usually carried out by changing the flow section $F(t)$. It is provided that the pressure and the area of the passage section changes according to the harmonic law (4). With the harmonic law, the amplitude, frequency and the phase angle are set, and the pressure change at the boundary is defined as:

$$
p=p_{\text {avg }}+p_{\max } \sin \left(2 \pi f \tau+\varphi_{0}\right),
$$

where $p_{\text {avg }}-$ average pressure in the mixing chamber, $\mathrm{Pa}$;

$p_{\max }$ - amplitude value of the pressure in the pulsator, $\mathrm{Pa}$;

$f$-ripple frequency, $\mathrm{s}^{-1}$;

$\varphi_{0}-$ initial phase.

To increase the ejection coefficient and maintain a high compression ratio in the ejector, the mixing chamber is made in the form of a cylindrical neck.

Based on this, it was found that the average pressure in the mixing chamber and the pressure amplitude in the pulsator determine the pressure in the pumped medium. Then the pressure formula of the pumped medium will look like this.

$$
p_{\text {avg }}=\frac{p^{*}}{\frac{\sqrt{(u+1)(1+u \theta+\vartheta)}}{1+1 / \alpha} \frac{q\left(\lambda_{1}\right)}{q\left(\lambda_{3}\right)}},
$$

where $p^{*}-$ total gas pressure, $\mathrm{Pa}$;

$u$ - ejection coefficient, $m_{2} / m_{1}$;

$m_{2}-$ mass of gas at the ejector outlet, $\mathrm{kg}$;

$m_{1}-$ gas mass at the ejector inlet, $\mathrm{kg}$;

$\theta$ - temperature ratio in the ejector;

$\alpha$-geometric coefficient, $\alpha=F_{1} / F_{2}$;

$F_{1}$ - output area of the active nozzle, $\mathrm{m}^{2}$;

$F_{2}$ - output area of the passive nozzle, $\mathrm{m}^{2}$;

$q(\lambda)$ - gas dynamic flow function.

Taking into account the formula (5), equation (4) will take the form:

$$
p_{\Sigma}=\frac{p^{*}}{\frac{\sqrt{(u+1)(1+u \theta+\vartheta)}}{1+1 / \alpha} \frac{q\left(\lambda_{1}\right)}{q\left(\lambda_{3}\right)}}+p_{\max } \sin \left(2 \pi f \tau+\varphi_{0}\right) \text {. }
$$

This formula makes it possible to describe and consider all the processes when using vacuum ejectors with a pulsator.

Preliminary calculations have shown that there is a boundary between velocities and pressures, where the influence of pulsation is not significant. At the same time, in a wide range of changes in these parameters, especially in the zone of supersonic currents, the ripple effect is significant.

It should be noted that in this case, the work of ejectors is considered in the one-dimensional theory of flow motion, since the complexity of mathematical solutions for two-dimensional flows makes these problems unsolvable. In addition, the results of the calculations performed on the one-dimensional theory are in good agreement with the experimental data.

The objects of experimental research were ejectors with pulsating motion of the active flow. The vacuum value was calculated using optimization methods developed at the Kazan State Agrarian University. The objective of the research was to determine and justify the main parameters of the ejector of the vacuum unit, as well as to provide experimental confirmation of the reliability of the research results. The main task of the experiments was to determine the most effective indicators of the ejectors, such as the degree of pressure increase, the ejection coefficient and the geometric parameter. Experimental studies were carried out according to the developed algorithm using a water ring pump VVN-0.25. Each parameter was measured 3 times, and their average value was taken. 


\section{Results and discussion}

As a result of the experimental studies, it was revealed that the operation of the pulsating ejector, in addition to the above parameters, is of great importance for the choice of a valve (shape and material) that provides an intermittent supply of the active flow.

As a result of joint mathematical processing of the experimental results, the dependences of the performance on the vacuum value were obtained for various ball materials (metal, rubber, and textolite):

$$
\begin{aligned}
Q_{M} & =6.8541+0.3659 \cdot P-0.0038 \cdot P^{2}, \\
Q_{r} & =4.6446+0.3874 \cdot P-0.0039 \cdot P^{2}, \\
Q_{t} & =3.0155+0.352 \cdot P-0.0037 \cdot P^{2} .
\end{aligned}
$$

Figure 2 shows the dependence of the supply of a water-ring vacuum pump with a pre-connected pulsating ejector $Q=f(P)$ on the value of the vacuum with metal, rubber and textolite balls of the valve interrupter of the active flow supply.

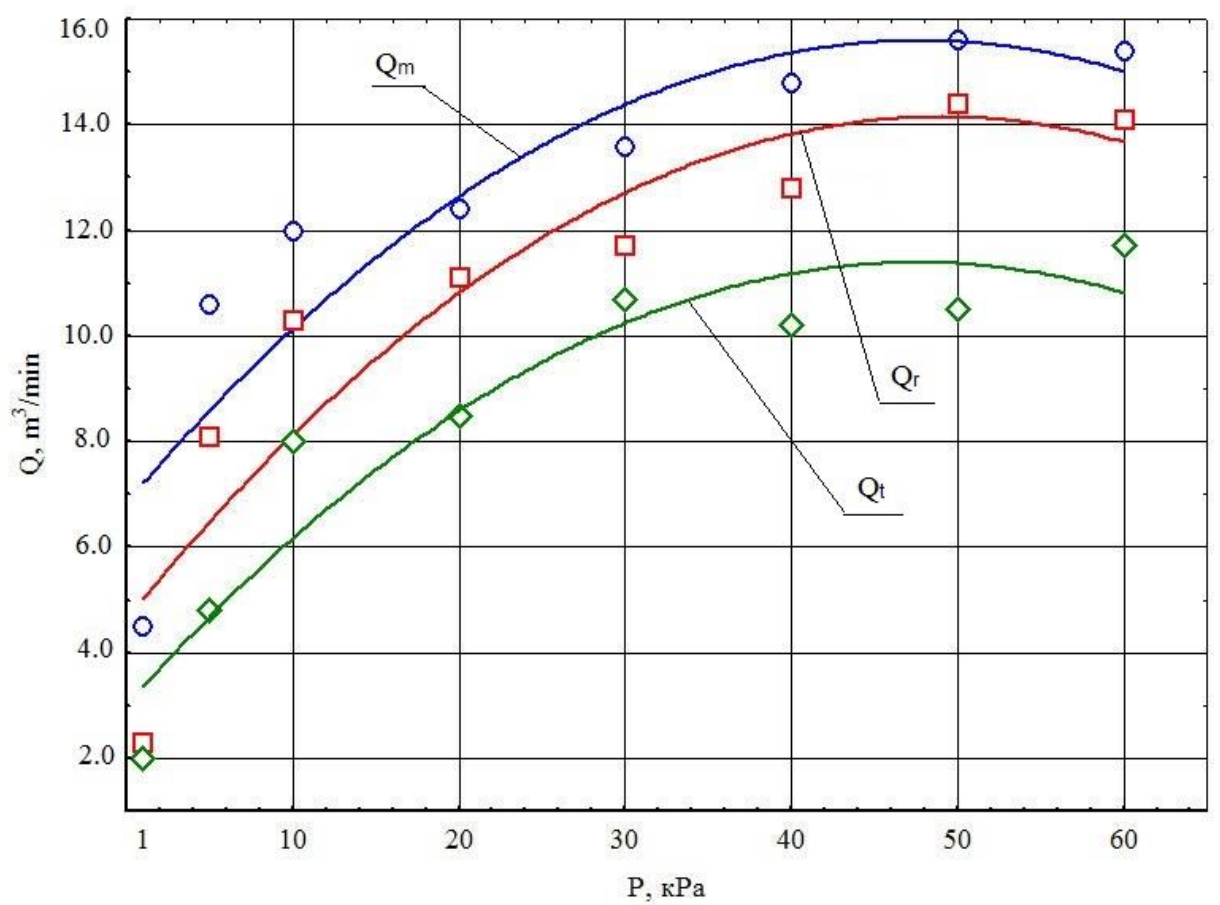

Fig. 2. Dependence of the flow of the ejector of a vacuum unit with a pulsating movement on the active flow with different flow interrupters (ball): $Q_{m}$ - for a metal ball;

$$
Q_{r} \text { - for a rubber ball; } Q_{t}-\text { for a textolite ball }
$$

The graph shows that for an ejector with a pulsating movement of the active flow, the recommended valve material is a metal ball.

As a result of joint mathematical processing of the experimental results, the dependences of the performance on the pulsation frequency for various vacuum values were obtained:

- for $P=20 \mathrm{kPa}$

$$
Q_{1}=13.515+0.0363 \cdot \omega-0.0003 \cdot \omega^{2},
$$

- for $P=30 \mathrm{kPa}$

$$
Q_{2}=13.965+0.0363 \cdot \omega-0.0003 \cdot \omega^{2},
$$

- for $P=50 \mathrm{kPa}$

$$
Q_{3}=14.265+0.0363 \cdot \omega-0.0003 \cdot \omega^{2},
$$

- for $P=60 \mathrm{kPa}$

$$
Q_{4}=13.366+0.0609 \cdot \omega-0.0004 \cdot \omega^{2} .
$$


Based on the obtained patterns, it was revealed that at a pulsation frequency of $90 \ldots 100 \mathrm{~min}^{-1}$, maximum performance is provided.

\section{Conclusions}

1. The pulsating jet vacuum pumping device is widely used in all industries and agriculture to transport various materials.

2. To increase the productivity of the technological process of transporting materials, an ejector with a pulsating movement of the active flow as part of a vacuum pumping device has been developed.

3. To describe the technological process of material transportation, using an ejector with a pulsating movement of the active flow, a theoretical dependence is presented.

4. In order to determine the most effective indicators of the ejectors, such as the degree of pressure increase, the ejection coefficient and geometric parameters, experimental studies were conducted.

5. The regularities of changes in the performance of a pulsating jet vacuum pumping device depending on the valve material and the magnitude of its pulsation are obtained.

6. It is revealed that for an ejector with a pulsating movement of the active flow, the recommended valve material is a metal ball, with a pulsation frequency of $90 \ldots 100 \mathrm{~min}^{-1}$.

7. As a result of the application of the proposed ejector with pulsating movement of the active flow, the transportation capacity will increase by $14.5 \%$.

\section{References}

[1] Fokin A.I., Tsoi Yu. A., Ziganshin B.G., Gayaziev I.N. Combined installation for milk cooling with the use of artificial and natural cold// Technika i oborudovanie dlya sela, 2015, No. 10, p. 11-12.

[2] Gomaa I.M., Kashapov I.I., Khaidarov R.R., Sabirov R.F., Khasanova F.F. Technological factors influence on the work efficiency of the feed grinder// BIO Web of Conferences. International Scientific-Practical Conference "Agriculture and Food Security: Technology, Innovation, Markets, Human Resources” (FIES 2019). 2020. C. 00233.

[3] Zinnatullin N.H., Ziganshin B.G., Nafikov I.M., Lushnov M.A., Zinnatullina G.N. Injection mixer of solid bulk materials// Bulletin of the Kazan State Agrarian University. 2017. Vol. 12. no. 3, pp. 68-71.

[4] Andrey Dmitriev, Bulat Ziganshin, Damir Khaliullin, Alexey Aleshkin Study of efficiency of peeling machine with variable deck // Engineering for rural development. 2020. C. 1053-1058.

[5] Belinsky A., Ziganshin B., Valiev A., Haliullin D., Galiev I. Theoretical investigation of increasing efficiency of combine harvester operation on slopes/// Engineering for Rural Development. 2019. C. 206-213.

[6] Ravil Ibyatov, Andrey Dmitriev, Bulat Ziganshin, Damir Khaliullin and Alsu Zinnatullina Mathematical modeling of the grain trajectory in the workspace of the sheller with rotating decks // BIO Web of Conferences 17, 00093 (2020).

[7] Ivanov, B.L., Ziganshin, B.G., Sharafeev, R.F., Sagbiev I.R. Teoriya nebulivanie zhidkami atomizkami [Theory of liquid atomization by injectors]. Vestnik Kazanskogo gosudarstvennogo agrarnogo universiteta [Bulletin of the Kazan State Agrarian University]. 2019. Vol. 14. No. 2 (53), pp. 95-99.

[8] Ziganshin B.G., Shogenov Yu.Kh., Lukmanov R.R. Technical solution for improving the efficiency of machine milking of cows // Bulletin of the Kazan State Agrarian University. 2016. Vol. 11. No. 1 (39), pp. 77-81.

[9] Lushnov M. A. Construction of a classification scheme and analysis of devices for heat treatment of semi-liquid feed mixtures// Agrarian science of the Euro-North-East. 2013. No. 5 (36), pp. 6568.

[10] Rudakov A.I., Nafikov I.R., Ivanov B.L. Improving the energy efficiency of freeze-drying of agricultural materials //Bulletin of the Kazan State Agrarian University. 2007, Vol. 2. No. 2 (6), pp. 101-105.

[11] Mustafin A.A., Ziganshin B.G. Analysis of the results of experimental studies of a two-rotor vacuum pump // Bulletin of the Kazan State Agrarian University. 2013, №1(27), pp. 63-68. 
[12] Mustafin A.A., Ziganshin B.G., Gainutdinov R.R., Gayaziev I.N. Method of calculation of a tworotor vacuum pump with involute engagement // Bulletin of the Kazan State Agrarian University, 2012, №1(23), Pp. 102-104.

[13] Ziganshin B.G., Gayaziev I.N., Kashapov I.I., Gainutdinov R.R., Nuriakhmetov T.R. To determine the design and technological parameters of a two-rotor vacuum pump // Vestnik Kazanskogo GAU, 2012. Vol. 7. No. 4 (26), pp. 75-78.

[14] Rudakov A.I., Asadullin N.M. Pulsing transportation of pseudoplastic liquids through pipes in animal husbandry// Vestnik Kazanskogo GAU. -2006, No. 4, pp. 62-67.

[15] Patent 2097606 of the Russian Federation, MKI 6 F 04 F 5/04. Pulsing ejector /A.I. Rudakov, N.M. Asadullin (Russia) . - No. 94009704/06. 15.03.94; publ. 27.11.97, byul. No. 33 - - 4 p.

[16] Abramovich G.N., Girshovich T.A. Theory of turbulent jets; ed. by G.N. Abramovich, Moscow: Nauka, 1984, 716 p. 